\title{
Application of Artificial Intelligence Techniques in Reactive Power/Voltage Control of Power System
}

\author{
Gehao Sheng* , Guangyu Tu and Yi Luo \\ Huazhong University of Science and Technology, Wuhan, Hubei 430074, China
}

\begin{abstract}
As one of the important constituents of power system automation, reactive power/voltage control possesses inherent characteristics of complexity, nonlinearity, inaccuracy and high requirement for control speed, parts of which are hard to be described by the traditional mathematical models or to be realized by routine control methods. The artificial intelligence (AI) techniques have intelligence feature which traditional method does not bear, so special attentions are paid to the application of AI techniques in reactive voltage control and a lot of results in this field are obtained. In this paper the main results and methods of applying the AI techniques, such as Expert System (ES), Artificial Neural Network (ANN), Fuzzy Theory (FT), Genetic Algorithm (GA) and Multi-Agent System (MAS), etc., to reactive voltage control in power systems are summarized, the respective application features of these techniques are analyzed and compared and some problems to be solved are pointed out.
\end{abstract}

\author{
KEYWORDS \\ Artificial intelligence \\ Reactive power \\ Voltage control \\ Power system
}

\section{Introduction}

Voltage and reactive power is closely related to the maintenance of the voltage level of the whole system, it must have sufficient reactive power to meet the system load demand for reactive power and reactive power compensation of losses and voltage control of power systems are mainly by controlling the reactive power generation, flow and consumption to achieve.

In recent years, a variety of intelligent control methods based on artificial intelligence technology has some conventional control do not have the intelligence, experience and knowledge can be introduced as experts, we can deal with uncertainty, with self-learning and knowledge acquisition function, appropriate nonlinear problems to, in the power system has been widely attention, get a lot of research, become a power system in an important area of research [1]. Power system voltage and reactive power control as an important part of power system automation,

Copyright () 2014 Gehao Sheng et al.

doi: 10.18686/esta.vli1.2

Received: March 12, 2014; Accepted: May 22, 2014; Published online: July 26, 2014

This is an open-access article distributed under the terms of the Creative Commons Attribution Unported License (http://creativecommons.org/ licenses/by-nc/4.0/), which permits unrestricted use, distribution, and reproduction in any medium, provided the original work is properly cited.

${ }^{*}$ Corresponding author: Huazhong University of Science and Technology, Wuhan, Hubei 430074, China. E-mail: shenggh1980@163.com power system control has inherent complexity, nonlinearity, inaccuracies and control requirements of real-time performance and other characteristics, so that some of them difficult to use traditional mathematical terms model and control methods to describe and implement, so researchers have begun to address these challenges by means of artificial intelligence methods, and promote intelligent control technology in the field of power system voltage and reactive power control is widely used. This article describes several major AI technologies such as expert systems (Expert System, ES), artificial neural networks (Artificial Neural Network, ANN), fuzzy theory (Fuzzy Theory, FT), genetic algorithm (Genetic Algorithm, GA) and Multi-Agent Systems (Multi-Agent System, MAS) and other basic concepts, and on this basis to related methods proposed in the literature in recent years, application of voltage and reactive power control are reviewed, analyzed and compared the characteristics of their applications, but also the problems raised, hope some inspiration for further research.

\section{Expert system in reactive power and voltage control}

Expert Systems (ES) is the older and more mature is a class of artificial intelligence techniques. Expert system is mainly made of knowledge and reasoning mechanism that reasoning based on specific areas of expert knowledge in a field provided to simulate a human expert decision-making process, to provide answers have expert level. At present, power system operation and control by experienced 
dispatchers complete with automated technology. This is because on the one hand the traditional numerical analysis method lacks heuristic reasoning ability, but also cannot be the accumulation of knowledge, on the other hand the complexity of the power system itself to make some necessary mathematical model and the state quantity are difficult to obtain, a simple numerical method is difficult to meet power system requirements, therefore, the introduction of empirical knowledge power experts in the power automation system is essential [1].

Expert System in Power System wide range of applications, in reactive power and voltage control also has more application of the results [2-11]. Expert systems are typically used in voltage and reactive power control is to experience or prior knowledge of voltage and reactive power control with rules that out, forming a knowledge base of expert system, and then according to the above rules by the reactive power and voltage in real time to strike a voltage change value control means to regulate the. Proposed the use of equivalent static networks and expert systems for large-scale power system voltage and reactive power control method, the expert system knowledge base includes upper and lower limits for each bus voltage, the controller controls the amount of each upper and lower limits, Each bus voltage and controls the amount of sensitivity and control flow logic rules and other information, the inference engine in accordance of the rules described in the knowledge base [2-8], in turn seek and select the most effective elimination of bus voltage deviation of the controller until the bus voltage recovery is set Value. The expert describes decision support system for a power system grid voltage control [5]. The system uses a centralized control strategy, control measures include adjusting the PV node voltage, transformers taps, and shunt capacitors, etc., depending on the control measures related to bus voltage control sensitivity and margin selection control step, the grid can correct abnormal voltage conditions. The text of the test results show that this method is more accurate than the decentralized control method, and the required control step less. The conventional methods and expert systems $[6,7]$ combining heuristic methods for system voltage and reactive power control. Where describes an integrated approach to running an auxiliary staff scheduling reactive voltage, the method using numerical method and expert system control variables to strike their respective advantages, can effectively eliminate the offset voltage, and improve the voltage characteristic minimum power loss [6]. Paper, according to the voltage on the control variables and the current-voltage curve of the sensitivity measurement of the effectiveness of the method, and describes its practical application in the control center. for more advanced hierarchical power system voltage control mode to optimize system partitioning method under study in the proposed algorithm partitioning basis based on the concept of electrical distance on the upward grade classification, and power systems based on expert knowledge automatic partitioning algorithm to adjust and optimize the results, to further ensure the rationality of the system partition and sub-regional voltage controllable [10].

For large substation voltage control, designed an expert system applied to substation voltage and reactive power automatic control [11]. The system is based on hierarchical thinking the whole control process is divided into multiple levels, their knowledge corresponding to the rule base into a tiered storage. Its inference engine and knowledge base each form module; simply the corresponding rule base target library can be modified for different substations, which has versatility. In addition, also describes a distribution system for reactive power control expert system [9].

In recent years, foreign and domestic have a lot of power system voltage and reactive power control related expert system put into trial operation or entered the practical stage of promotion, and achieved good results, but there are still some issues worthy of study and exploration: (1) When the large size of the system, when the rules are more complete inference speed is limited, so there are expert systems are mostly used for offline or online problemsolving part of the system analysis, and real-time control in terms of voltage and reactive power Application has just started, pending further study; (2) The existing expert systems lack effective learning mechanisms to deal with the limited capacity of the new situation, and poor fault tolerance, when the system fails or network structure, system parameters, reactive voltage equipment when the controller configuration changes, the result may not be obtained or give wrong results. How ANN, fuzzy reasoning and other artificial intelligence methods in combination to enhance self-learning ability and fault tolerance expert system is worthy of study; (3) Long construction period of large expert systems, knowledge acquisition more difficult and verification, to establish a comprehensive knowledge base maintenance more difficult, before the construction of the expert system must fully consider these issues.

\section{Artificial neural network in the application of the reactive voltage control}

Artificial Neural Network (ANN) is a simulation of human transmission and processing of the basic characteristics of information by a large number of simple imitation artificial neurons connected together in a certain way. Single artificial neuron is as an input to the output of non-linear relationship. The connection between them is important so that the combinations of ANN have complex nonlinear characteristics. Compared with ES, ANN is characterized by neurons and between them have to deal with weight problems to tacit knowledge and has the following advantages: information distributed storage, there is a strong fault tolerance; ability to learn, can be achieved knowledge of self-organization, to meet the requirements of different information processing; calculating between neurons with 
relative independence, ease of parallel processing, execution speed. It is precisely because there are strong nonlinear fitting ANN ability and self-learning ability, associative memory and has a robust and strong performance, making ANN power system for the presence of a large number of complex nonlinear systems are very large potential applications.

Currently, ANN in the automatic control of voltage and reactive power has been good application [12-17]. Proposed the use of two-stage ANN reactive power voltage control system. The first stage ANN system for detecting reactive power and voltage-limit node, the second stage gives ANN calibration strategy. In order to accelerate the convergence speed sample training, the paper also proposed an accelerated BP algorithm [16]. For a 34-node 50 branches to simplify the system tests show that the results are satisfactory [17]. For solving the reactive power with continuous Hopfield ANN model of the optimal distribution and economic dispatch, and were verified by numerical examples [12-15].

It will be used ANN substation automatic voltage control, also achieved good results. Constructed before feedforward neural network for voltage and reactive power control of decision-making, the input of the neural network comprises a main transformer through active and reactive power, high side and low side of the real-time voltage, output includes shunt capacitor switching state and the main transformer tap position, training samples of relevant historical data with power plant supervisory system $[12,14]$. This method has practical application in the power plant, the application can achieve better results indicate voltage and power factor control, and reduce the capacitor and primary transformer tap adjustment times. In addition to ANN for voltage and reactive power control decisions, but taking into account the substation reactive load is not as stable as active load, will ANN for reactive power load forecasting based on ANN presents a reactive power prediction and optimization strategy of combining integrated automatic voltage and reactive power control scheme. ANN prediction input reactive load of the first three weeks, the output is predicted reactive load. The text of the simulation results and compared the results of conventional methods based on field control, the advantages are obvious [15].

Currently, ANN applications there are still some problems, such as learning algorithm speed is generally slower, longer training time and difficult to converge or may converge to local minima. Some researchers working to improve the learning algorithm, greatly improved convergence performance. However, ANN some inherent flaws still not fully compensate, for example, the establishment of the model ANN lack of adequate theoretical guidance, when the system configuration changes need to add a new sample re-learning. In addition, the working process ANN is a black box, so despite ANN has some fault tolerance, but does not provide information to help run personnel inferred abnormal data, is not conducive to understanding its output. The way to use the advantages of ANN and overcome its shortcomings, to achieve better results, it is one of the important research directions ANN applications. In short, ANN applied research in the field of reactive power and voltage control of power system is still in the preliminary stage, there are a lot of artificial neural network model and algorithm with the characteristics have not been put to good use. With further research on the theory of ANN, this will have a broader application in voltage and reactive power control.

\section{The fuzzy theory in the application of the reactive voltage control}

Fuzzy Theory (FT) is the classic fuzzy set theory, and the introduction of linguistic variables and fuzzy logic approximate reasoning, complete with intelligent reasoning technology system. Fuzzy control is a practical method of controlling simulate human fuzzy reasoning and decisionmaking process and its control according to the known rules and data, is derived from the fuzzy input output fuzzy control including fuzzy, fuzzy reasoning and fuzzy judgment of three parts. With the development and improvement of fuzzy theory, some of the advantages of fuzzy control has been widely acknowledged, such as: suitable for dealing with uncertainty, imprecision and noise problems caused by; fuzzy knowledge to use language variables to express the experience of experts, closer to people's expression, easy to implement and express knowledge extraction; robust, impact of changes in the controlled object parameter fuzzy control not obvious. In recent years, increasing fuzzy theory in the study of power system applications and encouraging research results show the potential of fuzzy theory in solving power system problems.

System voltage and reactive power control by the power system degeneration, operating conditions, and network parameters often change the characteristics of power and reactive load under a number of conditions cannot be accurately given the impact is difficult to establish a precise mathematical model, in this case, blurred Study of voltage and reactive power control theory is introduced. There are many good fuzzy technology [18] currently used in the reactive planning in the system voltage and reactive power control, there are many related research reports [19-28]. Taking into account the actual operation of power system voltage and reactive power limits are not static, allowing a small amount of the more limited this case, the voltage limits fuzzy $[19,20]$, proposed a fuzzy linear programming method the goal is to determine the voltage required to maintain a minimum increase of reactive power. The example shows that the method more practical than the usual linear programming methods. According to those nodes for fault-point control of the formation of a strong local control of voltage and reactive power domain requirements 
[26], taking into account the strong and weak control itself has a certain degree of ambiguity, we propose a membership characterized by controlling the intensity, the next local emergency voltage and reactive power control method Fenwick with fuzzy clustering analysis. In the substation voltage control [27], proposed fuzzy control algorithm for the integrated control parallel capacitor switching and load tap tap switching, given fuzzy, fuzzy reasoning and fuzzy judgment implementation method. Comparison with traditional control algorithms show that the algorithm is small, easy to use single-chip real-time control, not only can effectively maintain the voltage within a certain range, and is conducive to rapid recovery fault system voltage stability, improved synchronization system stability.

In order to solve the system reactive power optimization control problems, there will be no power loss is minimized, to be balanced to improve voltage quality and reduce the number of multiple target control etc. [25], to limit these goals and voltage state variables fuzzy, a fuzzy multi-objective optimization methods. Real-time data acquisition in real regional power grid model for digital tested the effectiveness and flexibility of the method. It is used in conjunction linear programming optimization principle of fuzzy mathematics integrated approach [28], it gives no power optimization and linear programming related to each objective function optimal membership function problem to arrive at a satisfactory multi-objective problem the result.

Fuzzy technology exists self-learning ability is poor, fuzzy modeling difficulties and other issues, in order to overcome these shortcomings, there are many studies fuzzy theory combined with other artificial intelligence techniques, and achieved good results. Consider the case of reactive load cannot be accurately determined, the fuzzy theory and ANN, expert system to achieve optimal control of reactive power [21]. In this paper fuzzy neural network determines the membership of the control variables, fuzzy neural network input of reactive power load, the output fuzzy control variables, then strike through heuristic knowledge expert system the control variable to adjust the minimum amount of control mode, so that this method has better real-time performance. Digital simulation results show that the method can be applied to the online system voltage and reactive power control.

The main problem of fuzzy technology to control the presence of voltage and reactive power are: (1) Simple fuzzy information processing will cause the control accuracy of the system is reduced to improve the accuracy of the quantization levels will increase, causing the rule to expand the scope of the search, reduce the speed of decision-making; (2) Lack of systematic design approach, it is difficult to define precisely control objectives; (3) Control selection rules, select the domain theory, the definition of fuzzy sets, quantification, and other factors to select by trial and error method, which is sometimes complex systems to be ineffective. The fuzzy technology and expert systems, neural networks, genetic algorithms and integration of traditional control methods, can enhance the ability to deal with uncertainty, to some extent, solve the problem of fuzzy control simple existence, which is one of the focus in recent years, Fuzzy theory is applied also to the power voltage and reactive power control system has brought new vitality. With further study of fuzzy control theory, relevant research results will gradually move towards practical. In addition, the structure of fuzzy system identification has not yet formed a sound theory, how to choose the appropriate structure of fuzzy system voltage and reactive power control is also worthwhile research, the current preferred method is to control and optimization of fuzzy theory combined.

\section{Conclusion}

This article describes the expert systems, artificial neural networks, fuzzy theory, genetic algorithms and multi-agent system, artificial intelligence technology application in power system voltage and reactive power control. Artificial intelligence technology to achieve the above-mentioned ways from different voltage and reactive power control, have their own strengths, but also there is a corresponding shortage. How will these technologies combine to form an integrated intelligent control system, the control system can be complementary advantages of different methods, weaknesses, is the question now researchers concerned.

Currently, more comprehensive artificial intelligence technology has been applied in the power system. In terms of voltage and reactive power control, the paper also mentioned a number of related research results, but in general research in this area is not deep enough, nor needs the formation of systems analysis and design methods, practical research also to be further strengthened. From the current research trends and existing experience, combined with modern control theory fuzzy technology and ANN, combined with adaptive control and intelligent method of expert system has a great potential for application.

With further research on a variety of artificial intelligence, artificial intelligence technology and integrated intelligence technology will play a more important role in the field of electric power voltage and reactive power control system.

\section{Conflicts of interest}

These authors have no conflicts of interest to declare.

\section{Authors' contributions}

These authors contributed equally to this work.

\section{References}

1. Wu, J., \& Chen, W. (1998). Modern control technology applications in the power system. Chinese CSEE, 18(6).

2. Le, T. L., \& Negnevitsky, M. (1997). Network equivalents and expert system application for voltage and VAR con- 
trol in large-scale power system. IEEE Trans on Power Systems, 12(4).

3. Liu, C. C., \& Tomsovic, K. (1986). An expert system assisting decision-making of reactive power/voltage control. IEEE Trans on Power Systems, 1(3).

4. Le, T. L., Negnevitsky, M. (1995). Expert system application for voltage control and VAR compensation. Engineering Intelligent System, 3(2).

5. Singh, S. P. (1995). Sensitivity based expert system for voltage control in power system. International Journal of Electric Power \& Energy Systems, 17(6).

6. Martinez, J. L. (1995). A hybrid tool to assist the operator in reactive power/voltage control optimization. IEEE Trans on Power Systems, 10(2).

7. Hsu, Y. Y., \& Ho, K. L. (1992). Voltage control using a combined integer linear programming and rule-based approach. IEEE Trans on Power Systems, 7(2).

8. Keyhani, A. (1989). A rule based approach to construction of local network model for decentralized voltage control. Electric Power Systems Research, 16(1).

9. Salama, M. M., \& Chikhani, A. A. Y. (1992). An expert system for reactive power control of a distribution systems. IEEE Trans on Power Deli-very, 7(2).

10. Yu, W. Y. (1998). A power system based on expert knowledge of control voltage and reactive power optimization control of distributed hierarchical partitioning method. Chinese CSEE, 18(3).

11. Sun, S. X., \& Li, H., (1995). Large Substation Voltage and Reactive Power Automatic Control System Expert System. National Electric universities from professional Eleventh Annual Conference.

12. Hsu, Y. Y., \& Lu, F. C. A. (1998). Combined artificial neural network-fuzzy dynamic programming approach to reactive power/voltage control in a distributed substation. IEEE Trans on Power Systems, 13(4).

13. Hsu, Y. Y., \& Yang, C. C. (1994). A hybrid artificial neural network-dynamic programming approach for feeder capacitor schedule. IEEE Trans on Power Systems, 9(2).

14. Yang, D. L. (1999). Integrated automatic control based on ANN substation voltage and reactive power. Power System Automation, 23(13).

15. Du, H. W. (1995). Intelligent substation voltage and reactive power automatic control system. Universities nationwide electricity from professional Fifteenth Annual Symposium.

16. Hui, C. W., \& Li, Y. X. (1992). Application of artificial neural network voltage and reactive power control. China CSEE, 12(3).

17. Wen, F. S., \& Han, Z. X. (1992). Artificial neural network model of reactive power optimal distribution and economic dispatch. Chinese CSEE, 12(3).

18. Abdul-Rahman, K. H. \& Shahidehpour, S. M. (1994). Application of fuzzy sets to optimal reactive power planning with security constraints. IEEE Trans on Power Systems, $9(2)$.

19. Tomsovic, K. A. (1992). Fuzzy linear programming approach to the reactive power/voltage control problem. IEEE Trans on Power Systems, 7(1).

20. Bagriyanik, M., Aygen, Z. E. (1998). Fuzzy linear programming approach to the reactive power correction in electric power systems. Proceeding of APSCOM Peking.

21. Abdul-Rahman, K. H., \& Shahidehpour, S. M. (1995). Al approach to optimal VAR control with fuzzy reactive loads. IEEE Trans on Power Systems, 10(1).

22. Abdul-Rahman, K. H., \& Shahidehpour, S. M. (1993). A fuzzy-based optimal reactive power control. IEEE Trans on Power Systems, 8(2).

23. Su, C. T., \& Lin, C. T. (1996). A new fuzzy control approach to voltage profile enhancement for power systems. IEEE Trans on Power Systems, 11(3).

24. Lu, F. C., \& Hsu, Y. Y. (1994). Fuzzy dynamic programming approach to reactive power/voltage control in a distribution substation. IEEE Trans on Power Systems, 9(2).

25. Xu, G. G., \& Wang, X. (1998). Fuzzy Multi-Objective Approach To Optimal Voltage/Reactive Power Control. Proceeding of APSCOM-Peking.

26. Chun, L. B. (1995). The Method of Voltage and Reactive Power Fuzzy Set Theory Emergency Fenwick's. National electricity from professional colleges and universities on the 11 th annual meeting.

27. Duan, H. F., \& Li, X. Y. (1997). Voltage and reactive power based on fuzzy logic control strategy. Power system automation, 21(6).

28. Tan, J. C. (1990). Reactive power fuzzy math comprehensive excellent solution. Chinese CSEE, 10(Suppl). 